\title{
Vacancies in CuInSe(2): new insights from hybrid-functional calculations
}

Oikkonen, L E ; Ganchenkova, M G ; Seitsonen, A P ; Nieminen, R M

\begin{abstract}
We calculate the energetics of vacancies in $\mathrm{CuInSe}(2)$ using a hybrid functional (HSE06, HSE standing for Heyd, Scuseria and Ernzerhof), which gives a better description of the band gap compared to (semi)local exchange-correlation functionals. We show that, contrary to present beliefs, copper and indium vacancies induce no defect levels within the band gap and therefore cannot account for any experimentally observed levels. The selenium vacancy is responsible for only one level, namely, a deep acceptor level is an element of $(0 / 2-)$. We find strong preference for $\mathrm{V}(\mathrm{Cu})$ and $\mathrm{V}(\mathrm{Se})$ over $\mathrm{V}(\mathrm{In})$ under practically all chemical conditions.
\end{abstract}

DOI: https://doi.org/10.1088/0953-8984/23/42/422202

Posted at the Zurich Open Repository and Archive, University of Zurich ZORA URL: https://doi.org/10.5167/uzh-51641

Journal Article

Accepted Version

Originally published at:

Oikkonen, L E; Ganchenkova, M G; Seitsonen, A P; Nieminen, R M (2011). Vacancies in CuInSe(2): new insights from hybrid-functional calculations. Journal of Physics: Condensed Matter, 23(42):422202. DOI: https://doi.org/10.1088/0953-8984/23/42/422202 


\title{
Vacancies in CuInSe 2 : new insights from hybrid-functional calculations
}

\author{
L.E. Oikkonen ${ }^{1}$, M.G. Ganchenkova ${ }^{1}$, A.P. Seitsonen ${ }^{2}$, R.M. \\ Nieminen ${ }^{1}$ \\ ${ }^{1}$ COMP / Department of Applied Physics, Aalto University, P.O. Box 11000, \\ FI-00076 Aalto, Espoo, Finland \\ ${ }^{2}$ Physikalisch-Chemisches Institut, University of Zurich, Winterthurerstrasse 190, \\ CH-8057 Zürich, Switzerland
}

\begin{abstract}
.
The vacancy energetics in $\mathrm{CuInSe}_{2}$ are calculated with a hybrid functional (HSE06), which gives a better description of the band gap compared to (semi)local exchangecorrelation functionals. We show that, contrary to present beliefs, copper and indium vacancies induce no defect levels within the band gap and therefore cannot account for any experimentally observed levels. The selenium vacancy is responsible for only one level, namely, a deep acceptor level $\epsilon(0 / 2-)$. We find strong preference for $\mathrm{V}_{\mathrm{Cu}}$ and $\mathrm{V}_{\mathrm{Se}}$ over $\mathrm{V}_{\mathrm{In}}$ under practically all chemical conditions.
\end{abstract}




\section{Introduction}

$\mathrm{CuInSe}_{2}$ (CIS) is a semiconductor material mainly used as the absorber layer in thin-film solar cells. The solar cells incorporating CIS and its alloys, in particular $\mathrm{Cu}(\mathrm{In}, \mathrm{Ga}) \mathrm{Se}_{2}$ (CIGS), where In has been partly replaced by Ga, have outperformed all other thin-film candidates $[1,2]$, yet the fundamental reasons behind their outstanding performance are not well understood. The uncertainties can be traced back to the underlying defect physics. The operation and properties of the $p n$-junction-based solar cells are determined to a large extent by defects through doping. In the case of chalcopyrites such as CIS, doping is caused by intrinsic defects instead of impurity atoms as in other, more conventional semiconductors. Nevertheless, still after years of extensive research, the intrinsic defects responsible for self-doping in CIS have not been identified. This information would be essential in order to understand and control the doping behavior of the material and, thus, improve the solar cell performance in a systematic way.

The problems related to defect identification partly arise from the structural complexity of the material. Indeed, CIS exhibits a dozen of possible intrinsic defects, not to mention all the defect complexes that can be formed. This has complicated the experimental characterization of the material. Various studies employing methods such as photoluminescence, cathodoluminescence, or Hall measurements (for a review, see e.g. [3]), while providing information on the electronic defect levels and defect concentrations, have not shed light on the atomic structure of the defects. Recently, even the origin of some of these levels has been questioned [4, 5].

The missing links between electronic and atomic structures of the defects can be obtained from density-functional-theory (DFT) calculations, and several studies in this field have been published $[6,7,8,9,10]$. These studies have pinpointed defects and defect complexes (namely, $\mathrm{V}_{\mathrm{Cu}}, \mathrm{Cu}_{\mathrm{In}}+\mathrm{In}_{\mathrm{Cu}}$, and $2 \mathrm{~V}_{\mathrm{Cu}}+\mathrm{In}_{\mathrm{Cu}}$ ) which have remarkably low formation energies and are therefore expected to exist in CIS samples in considerable amounts. Transition levels corresponding to different defect charge states have also been predicted. For instance, considering the three vacancies, $\mathrm{V}_{\mathrm{Cu}}$ has been assigned with one shallow acceptor level $[6,9], \mathrm{V}_{\text {In }}$ with three transition levels [6], and $\mathrm{V}_{\text {Se }}$ with one shallow donor and one acceptor state within the band gap [9].

Experimental observations have been eagerly interpreted based on these theoretical findings: for example, the experimentally found $N 1$ defect level has been related to $\mathrm{V}_{\mathrm{Se}}$ or $\mathrm{V}_{\mathrm{Se}} \mathrm{V}_{\mathrm{Cu}}$ complex [11] and the lowest acceptor level has been associated with $\mathrm{V}_{\mathrm{Cu}}$ [6]. However, these theoretical results should be dealt with caution since they have been derived within the local-density approximation (LDA) formalism, which is known to underestimate semiconductor band gaps and thus leads to uncertainties in defect energetics. The underestimation is particularly severe for materials with strongly localized $d$-electrons such as CIS, where the repulsion between $\mathrm{Cu} 3 d$ and Se $4 p$ orbitals in the upper valence band is exaggerated to the point that the valence band maximum is pushed upwards and the LDA band gap is eliminated $[9,12,13,14,15]$. This problem has been attempted to solve with a posteriori correction methods, which, nevertheless, have 
not been proven to systematically improve the reliability of the results. Due to these ambiguities, the re-evaluation of defect energetics in CIS with more reliable methods is well motivated.

In this article, we show that the vacancies in CIS account for much fewer transition levels than what is presently believed: copper and indium vacancies create none, and selenium vacancy produces only one, a double acceptor. In the light of these new insights, the experimental results in this field should be reinterpreted. We have employed the range-separated hybrid functional HSE06 [16], which incorporates a fraction of nonlocal Hartree-Fock exchange. This gives an improved description of the CIS band gap as has been recently shown $[12,13,14]$.

\section{Computational details}

The calculations have been performed with the plane-wave code VASP [21, 22] using the projector-augmented wave method (PAW) [23]. The plane-wave basis set is truncated with a cutoff energy of $400 \mathrm{eV}$. The parameter $\alpha$ controlling the amount of Hartree-Fock exchange in the HSE06 functional is set to 0.25 as obtained from perturbation theory [24], and the range-separation parameter $\omega$ is set to 0.20 .

The fundamental bulk properties of CIS calculated with these settings are presented in Table 1. As can be seen, HSE06 improves the description of both the atomic and electronic structure: the values of all considered parameters are brought closer to experimental ones compared to semilocal PBE-GGA [17] results.

For defect calculations, one atom is removed from the supercell in order to create a vacancy, and the system is allowed to relax until the forces on each atom fall below $0.01 \mathrm{eV} / \AA$. The formation energies of the vacancies have been calculated as:

$$
E_{\mathrm{f}}=E_{\mathrm{tot}}^{\mathrm{defect}}-E_{\mathrm{tot}}^{\mathrm{bulk}}+\mu_{i}+q\left(E_{\mathrm{VBM}}+E_{\mathrm{F}}\right)
$$

where $E_{\text {tot }}^{\text {defect }}$ denotes the total energy of the supercell containing the vacancy, $E_{\text {tot }}^{\text {bulk }}$ is the total energy of a bulk supercell, $\mu_{i}$ is the chemical potential of the atom of type $i$ that has been removed in order to create the vacancy, $q$ is the charge state of the vacancy, $E_{\mathrm{VBM}}$ is taken as the energy corresponding to the valence band maximum

Table 1. Calculated lattice constants $a$ and $c$, band gap $E_{g}$, and $\mathrm{Cu} 3 d$ peak position for $\mathrm{CuInSe}_{2}$.

\begin{tabular}{lcccc}
\hline & $a(\AA)$ & $c / 2(\AA)$ & $E_{g}(\mathrm{eV})$ & $E_{3 d}(\mathrm{eV})$ \\
\hline This work: & & & & \\
PBE-GGA & 5.871 & 5.909 & 0.01 & -1.8 \\
HSE06 $(\omega=0.20)$ & 5.824 & 5.866 & 0.86 & -2.3 \\
HSE06 $(\omega=0.13)$ & $5.839[14]$ & $6.140[14]$ & $1.07[14]$ & - \\
Exp. & $5.781[18]$ & $5.809[18]$ & $1.04[19]$ & $-2.4[20]$ \\
\hline
\end{tabular}




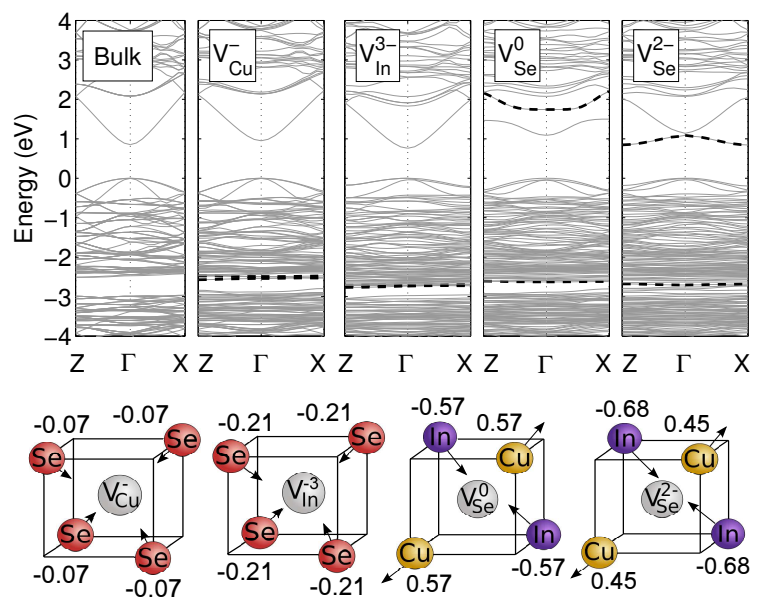

Figure 1. Band structures for bulk CIS, $\mathrm{V}_{\mathrm{Cu}}^{-}, \mathrm{V}_{\mathrm{In}}^{3-}, \mathrm{V}_{\mathrm{Se}}^{0}$, and $\mathrm{V}_{\mathrm{Se}}^{2-}$ calculated in a 64-atom supercell. The dashed lines illustrate the defect levels induced by the vacancy compared to the bulk band structure in each case. Shown below are the atomic relaxations (expressed in $\AA$ ) around each vacancy.

(VBM) of the bulk material, and $E_{\mathrm{F}}$ is the chemical potential for electrons defined relative to the VBM [25].

The transition energy is defined as the Fermi level position where the lowest-energy charge state changes from $q$ to $q^{\prime}$ :

$$
\epsilon\left(q / q^{\prime}\right)=\frac{E_{\text {tot }}^{\text {defect }}(q)-E_{\text {tot }}^{\text {defect }}\left(q^{\prime}\right)}{q^{\prime}-q}-E_{\mathrm{VBM}} .
$$

The chemical potentials $\mu_{i}$ can be defined with respect to the chemical potentials of the elemental solids $\mu_{i}^{0}: \mu_{i}=\mu_{i}^{0}+\Delta \mu_{i}$. In this case, $\mu_{i}^{0}$ 's are obtained from fcc $\mathrm{Cu}$, bct In, and trigonal Se. $\Delta \mu_{i}$ depends on the chemical composition of the material and is restricted to vary within the stability range of the chalcopyrite phase. The upper bound $\Delta \mu_{i}=0$ (corresponding to $i$-rich conditions) ensures that the elements do not precipitate into their elemental phases, while the lower bound marks the limit where competing phases such as $\mathrm{CuIn}_{5} \mathrm{Se}_{8}$ and $\mathrm{Cu}_{3} \mathrm{Se}_{2}$ start to form as described in [6, 8].

In order to take into account finite-size effects arising from the use of supercells in defect calculations, we have used finite-size scaling as discussed in [26], which requires performing a series of calculations using feasible different-sized supercells. In this work, we have considered the convergence of the configuration energy $E_{\text {conf }}=E_{\text {tot }}^{\text {defect }}-E_{\text {tot }}^{\text {bulk }}$ instead of the formation energy in order to avoid defining $E_{\mathrm{VBM}}$, which we can take from the bulk calculation after finite-size scaling [27]. We have employed supercells of 32 , 64, and 144 atoms, where the Brillouin zone sampling was done correspondingly using $2 \times 4 \times 2,2 \times 2 \times 2$, and $2 \times 2 \times 2 \mathrm{k}$-point sets. The $\Gamma$-point was used for the HartreeFock part. Due to the uncertainties involved in using only three values for the fit, we took the extrapolated value as an upper limit for $E_{\text {conf }}$. We repeated the extrapolation procedure with the PBE-GGA functional employing supercells up to 512 atoms and then added the so derived energy difference $\Delta E=E_{64}^{\mathrm{PBE}}-E_{\mathrm{inf}}^{\mathrm{PBE}}$ to the corresponding 
64-atom HSE06 result similarly to [28]. Since PBE-GGA is known to overestimate the static dielectric constant, we can expect the obtained value to be an appropriate lower limit for $E_{\text {conf }}$. We thus calculate the values of the configuration energies as an average of the lower and upper limits and, at the same time, obtain error estimates for them.

\section{Results and discussion}

The removal of either a $\mathrm{Cu}$ or In atom from the lattice has qualitatively similar implications on the atomic and electronic structure of CIS. This is expected since both cations have a similar chemical surrounding consisting of four nearest-neighbor Se atoms. With the creation of a cation vacancy, the Se atoms relax inwards nearly isotropically: $0.07 \AA\left(\sim 3 \%\right.$ of $\mathrm{Cu}$-Se bond length) in the case of $\mathrm{V}_{\mathrm{Cu}}$ and $0.21 \AA(\sim 8 \%$ of In-Se bond length) in the case of $\mathrm{V}_{\mathrm{In}}$ as shown in Figure 1. The relaxation is more pronounced for $\mathrm{V}_{\mathrm{In}}$ since the In atom is larger than $\mathrm{Cu}$.

Based on the careful analysis of the band structure (see Figure 1) and local density of states (LDOS), we conclude that no defect levels are induced within the band gap from either cation vacancy. Indeed, the only stable charge state is -1 for $\mathrm{V}_{\mathrm{Cu}}$ and -3 for $\mathrm{V}_{\mathrm{In}}$, corresponding to the fully occupied valence band. As seen from Figure 1, defect-induced single-particle levels appear deep in the valence band, at approximately $2.5 \mathrm{eV}$ below the VBM. These levels will always be occupied and therefore will not affect the doping behavior of CIS.

Our results strongly disagree with previous theoretical studies, which have found transition levels for both $\mathrm{V}_{\mathrm{Cu}}$ and $\mathrm{V}_{\mathrm{In}}$. A very shallow acceptor state $\epsilon(0 /-)$ has been attributed to $\mathrm{V}_{\mathrm{Cu}}[6,9]$, and $\mathrm{V}_{\mathrm{In}}$ has been claimed to exist in charge states spanning from 0 to $-3[6]$. These results have been derived within the LDA formalism and have required the application of additional a posteriori correction schemes for the band gap. For instance, Lany et al. [7, 8, 9, 10] used the band-edge correction to realign the VBM based on the $\mathrm{LDA}+\mathrm{U}$ method and then shifted the CBM to recover the experimental band gap. However, one should be careful when using this type of correction in cases where there is no band gap to begin with, since its application has been justified only for localized defects that are described well enough already at the LDA level [29]. Additional uncertainty is brought about by the choice of finite-size correction methods: the application of different potential alignment schemes result in qualitatively different outcomes ([7] vs. [9]). In another study [6], a small band gap was obtained with the linearized-augmented plane-wave method (LAPW), which was then stretched out with the scissor scheme. While the scissor scheme has been found to perform quite well in some cases after correcting for the finite-size errors [26], in this case, the supercell employed was too small (32 atoms) to represent accurately the total energies of systems including charged defects, even after local potential alignment.

In addition to the reasons outlined above, the discrepancies between our results and previous studies can stem from different ways of determining the position of the VBM. Notably, we have taken the value for $E_{\mathrm{VBM}}$ from the corresponding eigenvalue at the 


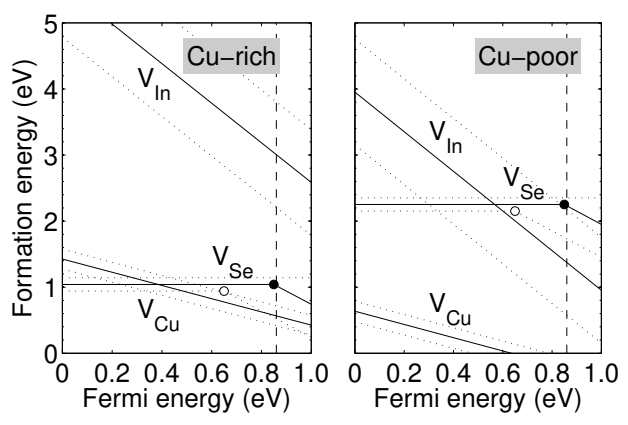

Figure 2. Vacancy formation energies as a function of the Fermi level. At the Cu-rich limit, the corresponding chemical potentials for In and Se are $\Delta \mu_{\mathrm{In}}=0 \mathrm{eV}$ and $\Delta \mu_{\mathrm{Se}}=-$ $1.21 \mathrm{eV}$. At the $\mathrm{Cu}$-poor limit, the chemical potentials are $\Delta \mu_{\mathrm{Cu}}=-0.79 \mathrm{eV}, \Delta \mu_{\mathrm{In}}=-$ $1.63 \mathrm{eV}$, and $\Delta \mu_{\mathrm{Se}}=0 \mathrm{eV}$. The dotted lines give error estimates for the formation energies at each Fermi level position. The dashed line marks the band gap obtained with HSE06.

$\Gamma$-point in bulk calculations. In contrast, a correction term has been applied in [6], and $\mathrm{LDA}+\mathrm{U}$ has been used to align the $d$-electron peak with photoemission spectra in [9]. Both ways shift the VBM downwards to such an extent that, for instance in the case of $\mathrm{V}_{\mathrm{Cu}}$, the $\epsilon(0 /-)$ transition state is revealed. This shift seems to be larger than what is obtained from HSE06 calculations, which is also known to push the VBM down [30].

Compared to the cation vacancies, $\mathrm{V}_{\mathrm{Se}}$ has quite a different effect on the electronic structure of CIS. In the chalcopyrite lattice, Se atom is surrounded by two $\mathrm{Cu}$ and two In atoms. With the removal of the Se atom, the nearest-neighbor In atoms relax towards each other, whereas the $\mathrm{Cu}$ atoms move away from each other (see Figure 1). The dangling bonds create a single-particle defect level inside the valence band (similar to $\mathrm{V}_{\mathrm{Cu}}$ and $\mathrm{V}_{\mathrm{In}}$ ) and another level above the VBM. These levels are clearly seen in Figure 1. For the neutral charge state, the unoccupied defect level appears even above the CBM, but the addition of two electrons shifts the level down to within the band gap. This lowering of the defect state is accompanied by enhanced lattice relaxation around the vacant site: $\mathrm{V}_{\mathrm{Se}}^{0}$ has a relaxation energy of $1.13 \mathrm{eV}$, whereas $\mathrm{V}_{\mathrm{Se}}^{2-}$ relaxes up to $1.55 \mathrm{eV}$ with the In atoms moving even closer to each other $(4 \%$ of In-Se bond length compared to $\mathrm{V}_{\mathrm{Se}}^{0}$ ). The charge density corresponding to the defect level is well localized between the In atoms.

Our results concerning the transition levels of $\mathrm{V}_{\mathrm{Se}}$ also differ from previously reported ones. Lany et al. have postulated that $\mathrm{V}_{\mathrm{Se}}$ is a negative-U defect having a shallow donor level $\epsilon(2+/ 0)$ and an acceptor level $\epsilon(0 / 2-)$ or $\epsilon(0 /-)$, depending on which type of potential alignment correction is applied [7, 9]. Contrary to their work, we find only the acceptor level, $\epsilon(0 / 2-)$, within the band gap at $0.85 \pm 0.20 \mathrm{eV}$ above the VBM. We have to dismiss the amphoteric behavior of $\mathrm{V}_{\mathrm{Se}}$ since we do not find the $2+$ charge state to be stable within the band gap irrespective of the initial geometry of the calculation setup. This discrepancy arises from the same reasons as discussed above for $\mathrm{V}_{\mathrm{Cu}}$. 
Figure 2 shows the vacancy formation energies as a function of the Fermi energy. The formation energies have been computed with Eq. 1. The range of the Fermi energy extends from the calculated VBM (corresponding to $0 \mathrm{eV}$ ) up to the experimental band gap $(1.04 \mathrm{eV})$.

Based on our results, $\mathrm{V}_{\mathrm{Cu}}$ is the most prominent out of the three vacancies in CIS as it has the lowest formation energy under practically all conditions. The low $\mathrm{V}_{\mathrm{Cu}}$ formation energy is in line with the experimental observation that CIS is easily grown $\mathrm{Cu}$-poor [3] as well as previously published theoretical studies [6, 8, 9, 14]. Further, it leads to Fermi level pinning when the formation energy drops below zero. In our case, depending on the chemical conditions, the Fermi pinning level can vary from $0.62 \pm 0.15$ $\mathrm{eV}$ up to the gap value, which is compatible with experimentally determined Fermi pinning levels of around $0.85 \mathrm{eV}$ for $\mathrm{Cu}$-poor films reported in [31].

Concerning the two other vacancies, $\mathrm{V}_{\mathrm{Se}}$ has a formation energy comparable to $\mathrm{V}_{\mathrm{Cu}}$ in $\mathrm{Cu}$-rich conditions but becomes energetically less favorable under $\mathrm{Cu}$-poor conditions. In contrast, $\mathrm{V}_{\text {In }}$ exhibits high formation energies almost irrespective of chemical conditions, implying that it will contribute insignificantly to vacancy concentrations in CIS material. As a whole, the relative magnitudes of the formation energies of the different vacancies follow quite well previously published results [6, 9] although the values obtained in this study are larger.

\section{Conclusion}

In summary, we have presented vacancy energetics for CIS obtained from hybridfunctional calculations. We have shown that, contrary to present beliefs, $\mathrm{V}_{\mathrm{Cu}}$ and $\mathrm{V}_{\mathrm{In}}$ induce no defect ionization states within the band gap and therefore cannot account for any experimentally observed levels. $\mathrm{V}_{\mathrm{Se}}$, on the other hand, is responsible for only one level, a double acceptor level at $0.85 \pm 0.20 \mathrm{eV}$ above the VBM. Because of its relatively low formation energy, $\mathrm{V}_{\mathrm{Cu}}$ is expected to exist in CIS material in substantial concentrations. Overall, there is strong preference for $\mathrm{V}_{\mathrm{Cu}}$ and $\mathrm{V}_{\mathrm{Se}}$ over $\mathrm{V}_{\mathrm{In}}$ under practically all chemical conditions. According to previous theoretical studies, vacancies in CIS can form larger aggregates and complexes with antisites [6, 7]. The energetics of these complexes should be now re-examined with a more accurate procedure.

\section{Acknowledgments}

This work has been supported by Academy of Finland through its Center of Excellence program (2006-2011). We are grateful to Center of Scientific Computing, Espoo, for computational resources.

\section{References}

[1] M. Kemell, M. Ritala, and M. Leskelä, Crit. Rev. Solid State Mat. Sci. 30, 1 (2005).

[2] I. Repins et al., Prog. Photovoltaics 16, 235 (2008). 
[3] S. Siebentritt, M. Igalson, C. Persson, and S. Lany, Prog. Photovoltaics 18, 390 (2010).

[4] T. Eisenbarth, T. Unold, R. Caballero, C.A. Kaufmann, and H.-W. Schock, J. Appl. Phys. 107, 034509 (2010).

[5] U. Reislöhner, H. Metzner, and C. Ronning, Phys. Rev. Lett. 104, 226403 (2010).

[6] S.B. Zhang, S.H. Wei, A. Zunger, and H. Katayama-Yoshida, Phys. Rev. B 57, 9642 (1998).

[7] S. Lany and A. Zunger, Phys. Rev. B 72, 035215 (2005).

[8] C. Persson, Y.J. Zhao, S. Lany, and A. Zunger, Phys. Rev. B 72, 035211 (2005).

[9] S. Lany and A. Zunger, J. Appl. Phys. 100, 113725 (2006).

[10] S. Lany and A. Zunger, Phys. Rev. Lett. 100, 016401 (2008).

[11] M. Igalson, A. Urbaniak, and M. Edoff, Thin Solid Films 517, 2153 (2009).

[12] J. Paier, R. Asahi, A. Nagoya, and G. Kresse, Phys. Rev. B 79, 115126 (2009).

[13] J. Vidal, S. Botti, P. Olsson, J.-F. Guillemoles, and L. Reining, Phys. Rev. Lett. 104, 056401 (2010).

[14] J. Pohl and K. Albe, J. Appl. Phys. 108, 023509 (2010).

[15] H. Xiao, J. Tahir-Kheli, and W. Goddard, J. Phys. Chem. Lett. 2, 212 (2011).

[16] J. Heyd, G. Scuseria, and M. Ernzerhof, J. Chem. Phys. 118, 8207 (2003).

[17] J.P. Perdew, K. Burke, and M. Ernzerhof, Phys. Rev. Lett. 77, 3865 (1996).

[18] W. Paszkowicz, R. Lewandowska, and R. Bacewisz, J. Alloy. Compd. 362, 241 (2004).

[19] Ariswan, G. El Haj Moussa, F. Guastavino, and C. Llinares, J. Mater. Sci. Lett. 21, 215 (2002).

[20] T. Löher, A. Klein, C. Petterkofer, and W. Jaegermann, J. Appl. Phys. 81, 7806 (1997).

[21] G. Kresse and J. Furthmüller, Phys. Rev. B 54, 11169 (1996).

[22] G. Kresse and J. Furthmüller, Comp. Mater. Sci. 6, 15 (1996).

[23] G. Kresse and D. Joubert, Phys. Rev. B 59, 1758 (1999).

[24] J. Perdew, M. Ernzerhof, and K. Burke, J. Chem. Phys. 105, 9982 (1996).

[25] C. Van de Walle and J. Neugebauer, J. Appl. Phys. 95, 3851 (2004).

[26] C. Castleton, A. Höglund, and S. Mirbt, Model. Simul. Mater. Sci. Eng. 17, 084003 (2009).

[27] K. Laaksonen, M. Ganchenkova, and R. Nieminen, J. Phys.: Condens. Matter 21, 015803 (2009).

[28] P. Agoston et al., J. Appl. Phys. 108, 053511 (2010).

[29] A. Alkauskas, P. Broqvist, and A. Pasquarello, Phys. Rev. Lett. 101, 046405 (2008).

[30] J. Lyons, A. Janotti, and C. Van De Walle, Appl. Phys. Lett. 95, 252105 (2009).

[31] A. Klein and W. Jaegermann, Appl. Phys. Lett. 74, 2283 (1999). 